\title{
Protective Mechanism of Xiyin Liangxue Decoction on Immunoglobulin A Nephropathy Rats
}

\author{
JING WEI, MING LI, XIAOFANG YAN, CHUN LIU AND ZEMEI ZHOU* \\ Department of Nephrology, Chongqing General Hospital, 104 Loquat Hill Street, Yuzhong District, Chongqing, 400014 China
}

Wei et al.: Mechanism of Xiyin Liangxue Decoction on IgA Nephropathy Rats

\begin{abstract}
Immunoglobulin A nephropathy is the most common glomerular disease in China, accounting for about $20 \%$ to $47 \%$ of primary glomerular diseases. Xiyin liangxue decoction is a common experience in the treatment of immunoglobulin A nephropathy in recent years. The research of this formula in the clinical treatment of immunoglobulin A nephropathy has achieved obvious curative effect. This article aims to study the protective mechanism of xiyin liangxue decoction on immunoglobulin A nephropathy rats and analyzes and compares the advantages of xiyin liangxue decoction and western medicine in the treatment of immunoglobulin A nephropathy rats with Sprague dawley nephropathy rats. The results of the study showed that after treatment of immunoglobulin A nephropathy rats with xiyin liangxue decoction, the renal function of the rats in the treatment group was significantly improved, the expressions of alanine aminotransferase and aspartate aminotransferase increased by $19 \%$ and $5 \%$ and the expressions of albumin and globulin increased by $19 \%$ and $25 \%$. In addition, in the treatment group, the number of urine red blood cells, urine protein and urine mannose binding lectin decreased by $29 \%, 13 \%$ and $25 \%$, respectively. The urine volume of rats in the high dose group was significantly reduced by $13 \%$ and the expressions of blood urea nitrogen and blood creatinine increased $22 \%$ and $8 \%$ respectively. After treatment with xiyin liangxue decoction, the cure rate of experimental immunoglobulin $A$ rats reached $24 \%$ and $33 \%$ had significant improvement. It can be seen that xiyin liangxue decoction has a good clinical treatment effect on immunoglobulin A nephropathy rats.
\end{abstract}

Key words: Xiyin liangxue decoction, IgA nephropathy, kidney protection, mechanism research

Immunoglobulin A nephropathy (IgAN) is the most common glomerular disease in China, accounting for $20 \%$ to $47 \%$ of primary glomerular diseases, and is characterized by the deposition of low glycosylated IgA1 in the glomeruli. In recent years, data show that the incidence of end stage renal disease (ESRD) has increased year by year and about $30 \%-40 \%$ of patients have entered ESRD within $10 \mathrm{y}$. This is the first reason for primary glomerular disease to require renal replacement therapy. IgA nephropathy is characterized by kidney biopsy pathology, which shows the deposition of immune complexes based on IgA in the mesangial area, while histologically, the glomerular area shows hyperplasia based on IgA. Currently, there is no established method for the treatment of IgA nephropathy. Although there are angiotensinconverting enzyme inhibitors (ACEI), angiotensin receptor blockers (ARB), afforded and other drugs with good curative effects, the side effects of these drugs limit the universality of their clinical applications. Clinical studies have shown that integrated traditional

*Address for correspondence E-mail: 276910637@qq.com
Chinese and Western medicine treatment is superior to simple western medicine, especially in controlling recent clinical symptoms, improving patients' quality of life and improving poor prognosis.

\section{MATERIALS AND METHODS}

Xiyin liangxue decoction is an experimental method for treating IgA nephropathy in recent years and it has a good effect on improving the clinical symptoms and urine test results of patients with IgA nephropathy ${ }^{[1]}$. Xiyin liangxue decoction has the effect of nourishing liver and kidney, cooling serum heat. Angelica and Salvia militarize in the prescription have the effect of nourishing blood and promoting blood circulation.

\footnotetext{
This is an open access article distributed under the terms of the Creative Commons Attribution-NonCommercial-ShareAlike 3.0 License, which allows others to remix, tweak, and build upon the work non-commercially, as long as the author is credited and the new creations are licensed under the identical terms
} 
Ophiopogon japonicus, ginseng and sea anemone can clear away heat and dryness, cicada insects and aconite scattered wind, reduce itching, Licorice root clears away heat and detoxifies, coordinating various drugs ${ }^{[2]}$. In addition, Xiyin liangxue decoction has analgesic, anti-inflammatory, anti-viral, sedative, anti-convulsant, etc. Ginseng and Ophiopogon japonicus have analgesic, antipyretic, anti-inflammatory, antibacterial and heart enhancing effects and anemone has anti-pathogenic microorganisms, antipyretic, hypoglycemic and antiplatelet aggregation effects ${ }^{[3]}$. This shows that Xiyin liangxue decoction is a good prescription for the treatment of IgA nephropathy, which deserves attention and research.

This article discusses the research on the protective mechanism of Xiyin liangxue decoction on the kidneys of IgA nephropathy rats. Nagata investigated the basic ingredients of Xiyin liangxue decoction, fed Chinese herbal medicine to IgA nephropathy rats and compared the efficacy of Xiyin liangxue decoction, while feeding animals with $300 \mathrm{~g} / \mathrm{kg}$ feed for $28 \mathrm{~d}$; it was found that IgA nephropathy fed Xiyin liangxue decoction. The $\mathrm{pH}$ value of the cecum of the rat was significantly reduced and its immunoglobulin A (IgA) and ammonia nitrogen concentrations were significantly higher ${ }^{[4]}$. Han proposed that Xiyin liangxue decoction has a very important and significant effect on the kidney protection of IgA nephropathy rats. It is believed that Xiyin liangxue decoction is more effective in treating IgA nephropathy rats than western medicine, although it takes a longer time, but with fewer side effects ${ }^{[5]}$. Turkez examined the potential toxicity of various concentrations $(0-1000 \mathrm{ppm})$ of LTT NP $(<100 \mathrm{~nm})$ in cultured primary rat kidney cells through 3-(4,5-dimethylthiazole-2Base) 2,5-diphenyltetrazolium bromide (MTT) determination and lactate dehydrogenase (LDH) release to detect cell viability and found that all aspects of kidney disease rats fed Xiyin liangxue decoction are better $^{[6]}$. Shinoda pointed out that the key to treating IgA nephropathy rats is to reduce side effects and strengthen prognosis management. It is difficult for modern western medicine to obtain curative effects while reducing the occurrence of toxic and side effects. This can be solved from ancient Chinese prescriptions ${ }^{[7]}$.

In the study of the protective mechanism of Xiyin liangxue decoction on IgA nephropathy rats, this paper summarizes and analyzes the research experience and achievements of a large number of predecessors and has made some innovations in the research content and research methods, specifically the following points: first, this article has studied in detail the role of renal podocytes in IgA nephropathy and found that podocytes have abnormal expression in more than $55 \%$ of IgA nephropathy cases. Second, this article uses the method of field investigation in the study, taking Sprague Dawley (SD) nephropathy rats as the research object, effectively comparing the different treatment effects of Xiyin liangxue decoction and other western medicines on IgA nephropathy, showing the advantages of Xiyin liangxue decoction. Third, this article uses the SPSS 15.0 statistical software package for the first time to process the data obtained by the experiment, ensuring the accuracy and reliability of the conclusion when comparing the research data.

\section{Feasibility of xiyin liangxue decoction in treating IgA nephropathy:}

Epidemiology and pathogenesis of IgA nephropathy: The diagnostic criteria for IgA nephropathy are kidney biopsy pathology, which is characterized by kidney biopsy showing the presence of IgA based immune complexes in the renal glomerular area and histologically showing IgA based hyperplasia in the kidney's glomerular area. There is no name for IgA nephropathy in Chinese medicine records, but there are corresponding diseases such as "edema", "urinary blood", "fatigue" and "turbidity", of which edema is the most common name. The edema record in the book "Spiritual Zone" is the earliest description of edema in China's existing medical literature, describing the specific clinical manifestations of the occurrence and development of edema. Images of symptoms of edema are described as "slightly swollen eyes and newly laid shape", while edema and bloating of extremities are symptoms of edema. The clinical manifestations of IgA are diverse, and it is difficult to determine the clinical symptoms of $\operatorname{IgA}$ nephropathy related to the pathological grade of IgA, but studies have shown that individual differences are large ${ }^{[8,9]}$.

Research on the pathogenesis of modern IgA nephropathy: IgA nephropathy is a disease that is caused by a combination of factors. Many modern scholars believe that there are three main immune systems in the human body that are closely related to the disease, namely the bone marrow system, the blood system and the kidney system. So far, the most studied factor is the influence of genetics and immunity on the pathogenesis and development of IgA nephropathy. Studies have shown that the genetic factors of IgA nephropathy may appear in more complex gene expression forms. 
Therefore, the role of genetic factors in the occurrence, development and prognosis of IgA nephropathy needs further study. Secondly, in terms of immunity and blood, studies have shown that about $45 \%$ of patients with IgA nephropathy have increased serum IgA content. In these elevated serum IgA, the IgA1 subtype is mainly polymerized, usually immunized with large molecules the form of the complex appears (IGA-IC) ${ }^{[10]}$. In addition, as far as pathology is concerned, although IgA nephropathy is a glomerular disease characterized by hyperplasia of the mesangial, IgA immune complexes in the mesangial area or IgA-based IgA immune complex particles. However, so far, most experts and scholars believe that the factors leading to poor prognosis of IgA nephropathy include a large amount of proteinuria, renal insufficiency, hypertension and other diseases. Therefore, how to reduce urine protein, protect renal function, and control blood pressure is the treatment of IgA nephropathy the essential.

\section{Insufficiency of modern medical treatment plan} for IgA nephropathy and availability of xiyin liangxue decoction: At present, the principles of modern medical treatment for IgA nephropathy mainly include the prevention and treatment of infections, control of blood pressure, reduction of proteinuria and protection of the kidneys, avoiding overwork, avoiding the use of dehydrating drugs and nephrotoxic drugs, and regular review. Some scholars in Europe and America suggested the use of fish oil, but because of its uncertain efficacy, it is not clinically recognized. In addition, one of the commonly used methods in western medicine is to use glucocorticoids to treat IgA nephropathy. The application of glucocorticoids and immunosuppressants in IgA nephropathy is mainly through the suppression of overactive autoimmunity, thereby reducing the deposition of immune complexes in the glomeruli, thereby reducing the damage of the glomerular filtration barrier. However, the side effects of IgA nephropathy treated with glucocorticoids are also very prominent, among which the common ones are induced or aggravated infection, femoral head necrosis, bone marrow suppression, liver injury, peptic ulcer ${ }^{[11]}$. Therefore, when patients with IgA nephropathy need to use such drugs for a long time, they should not take calcium supplements, stomach protectors, liver protectants and other drugs at the same time, otherwise serious and serious life threaten may occur. For patients, the use of some western medicine not only means bearing a high risk, but also means bearing a huge financial burden. Therefore, it is undeniable that traditional Chinese medicine (TCM) can effectively reduce the side effects of glucocorticoids and immunosuppressants and has the advantages of reducing toxicity and improving the therapeutic effect of Western medicine.

Xiyin liangxue decoction is a well-known TCM experience in treating chronic kidney disease. Its efficacy is mainly to nourish qi and nourish yin, strengthen spleen and kidney. From the theoretical analysis of TCM, Xiyin liangxue decoction is mainly for correcting, which is completely different from the mechanism of TCM treatment of nephropathy in two aspects. The main mechanism of Xiyin liangxue decoction to treat kidney disease is to nourish yin and nourish yin, so as to adjust the balance of yin and yang of the human body as a whole, adjust the body's immune function and finally achieve the purpose of restoring righteousness, removing evil spirits and curing diseases.

Modern research has shown that the unique immune regulation function of TCM in the treatment of autoimmune diseases is the main mechanism of its therapeutic effect, which is also one of the distinguishing characteristics of TCM. Modern pharmacological studies have found that the Zoonosis Pilosa polysaccharide in Xiyin liangxue decoction can regulate the body's immune system, promote the proliferation of normal splenic lymphocytes and enhance the production of antibodies in normal rats. In addition, studies have shown that Xiyin liangxue decoction can delay the progress of $\operatorname{IgA}$ nephropathy by regulating the imbalance of $\mathrm{Th} 1$ and $\mathrm{Th} 2$ in IgA nephropathy model rats $^{[12]}$. It can also promote humoral immunity in immunocompromised mice, reduce urinary protein excretion in mouse models of nephropathy, and improve pathological changes such as glomerular podocyte fusion. Therefore, according to the pharmacological analysis of modern single drugs, Xiyin liangxue decoction plays a certain role in regulating immune function, anti-inflammatory and diuretic. It can be speculated that in addition to protecting podocytes, the treatment of IgA nephropathy by TCM may be mainly related to its role in immunoregulatory function.

\section{Study on the protective mechanism of xiyin liangxue decoction on IgA nephropathy rats:}

\section{Experimental objects and experimental materials:} Animals used in the experiment; a total of 40 male SD rats were selected for the experiment, and the individual mass distribution was between $180 \mathrm{~g}$ and $220 \mathrm{~g}$. All the rats were provided by Shanghai Xiyu Biak 
Experimental Animal Co., Ltd. The animal certificate number: 2008001666586. The certificate number is: SCXK (Shanghai) 2013-0016. All rats are kept in an experimental animal center. The feeding conditions are: room temperature is maintained at $20^{\circ} \sim 24^{\circ}$, relative humidity is maintained at $50 \% \sim 80 \%$, during the breeding period, experimental rats can freely drink water and eat, without limiting its other activities. Feeding: the dosage is 5 times the adult dose $(\mathrm{kg} / \mathrm{g})$ as the low dose and 10 times the adult dose $(\mathrm{kg} / \mathrm{d})$ as the high dose. Use distilled water to prepare a proportion of each milliliter of liquid equivalent to the amount of herbal medicine. $0.3 \mathrm{~g}$ and $0.6 \mathrm{~g}$, supplemented by a homogenizer to promote the dissolution of herbs, then the solution is bottled and sealed and placed in a refrigerator at $4^{\circ}$ to be kept for use.

Experimental reagents and instruments: Xiyin liangxue decoction Chinese medicine granules (provided by Jiangyin Tianjin Pharmaceutical Co., Ltd. in Jiangsu Province), Benazepril (Beijing Novartis Pharmaceutical Co., Ltd.), Chinese medicine granules (Sichuan New Green Pharmaceutical Technology Development Limited Company), bovine serum albumin (bovine serum albumin, BSA), lipopolysaccharide (LPS) provided by Sigma, Carbon tetrachloride $\left(\mathrm{CCL}_{4}\right)$, castor oil (Shanghai McLean Biochemical Technology Co., Ltd.), lab reagent ( Lot No. 022/051/1, Shanghai Shinning Desai Diagnostics Co., Ltd.), aspartate aminotransferase (AST) reagents (batch number 26023/962077/4, Shanghai Shinning Desai Diagnostics Co., Ltd.). Experimental instruments mainly include: Hitachi 7020 automatic biochemical analyzer, ZEISS frozen microtome, paraffin tissue microtome, KUDOS ultrasonic cleaning instrument, electric thermostatic water tank, plate washer, automatic enzyme labeling instrument, electronic balance, drug balance with shelf, desktop multi-purpose centrifuge, low-speed large-capacity multi-tube centrifuge, fluorescence microscope, ultraviolet spectrophotometer, etc. The other experimental instruments used in this experiment are shown in Table 1.

Preparation of experimental drugs in groups: $40 \mathrm{mg} / \mathrm{ml}$ BSA solution: weigh $20 \mathrm{~g} \mathrm{BSA}$, add $500 \mathrm{ml}$ of physiological saline to the BSA solution and prepare other solutions according to this ratio and store in a refrigerator at $4^{\circ}$ for future experiments. Preparation of $\mathrm{CCL}_{4}$ solution: take $30 \mathrm{ml}$ of castor oil and $410 \mathrm{ml}$ of castor oil respectively, mix according to this ratio, then immediately shake the mixture and store it in a refrigerator at $4^{\circ}$ for future experiments.

Preparation of LPS solution: take $10 \mathrm{mg}$ of LPS and dissolve in LML sterile saline. After the LPS sterile saline solution is completely dissolved, use a pipette to take $500 \mu 1$ of LPS solution, add $100 \mathrm{ml}$ of sterile saline solution, let it mix thoroughly and use it immediately. When not in use, put it in a $4^{\circ}$ refrigerator for later use experimental use. Preparation of low dose xiyin liangxue decoction: Take a total of $220 \mathrm{~g}$ of xiyin liangxue decoction granules, dissolve them in $500 \mathrm{ml}$ of distilled water, mix the two according to this ratio and seal them in a refrigerator at $4^{\circ}$ for future use.

Preparation of high-dose xiyin liangxue decoction: dissolve $440 \mathrm{~g}$ of xiyin liangxue decoction granules in $500 \mathrm{ml}$ of distilled water, mix according to this ratio, seal and store in a refrigerator at $4^{\circ}$ for future use.

\section{Experimental method:}

The 40 SD rats prepared for the experiment were raised in the clean animal room of a certain animal experiment research center. The rats were fed for a period of time and brought to the laboratory. After weighing in the laboratory, the rats were randomly divided into 4 groups, with 10 rats in each group. The first is the treatment of rats in the model group: BSA solution is used to supplement the stomach of the rats every other day. The stomach supplementation of the rats in each group continues for $8 \mathrm{w}$ at the same time and then the rats are injected with $\mathrm{CCL}_{4}$ solution $(0.6 \mathrm{ml} / \mathrm{w})$, more than $9 \mathrm{w}$, during the combined use of LPS solution $(\mathrm{mg} / 0.05)$, tail vein injection in the $6^{\text {th }}$ and $8^{\text {th }} \mathrm{W}$, each rat a total of 2 times. Treatment of normal control rats: from the $1^{\text {st }} \mathrm{W}$ of the experiment, rats were given normal saline $(1 \mathrm{ml} / 100 \mathrm{~g})$ every other day for a total of $8 \mathrm{w}$, and then the rats were injected subcutaneously with $0.9 \%$ sodium chloride solution $(0.4 \mathrm{ml} /$ tablet, once a w for $9 \mathrm{w}$ ) and at the 6th and 8th $\mathrm{w}$, rats were injected with $0.9 \%$ sodium chloride (Nacl) $0.2 \mathrm{ml} /$ branch through

TABLE 1: OTHER EXPERIMENTAL INSTRUMENTS FOR THIS EXPERIMENT

\begin{tabular}{lcc}
\hline Name & Type & Source \\
\hline PCR Gene Amplifier & ZEISS HM505E & Bio-Rad, American \\
Ultra-thin slicer & EMUC7 & LEICA, Germany \\
Critical point dryer & K850 & EMITECH, Britain \\
Vacuum spraying instrument & MODEL E-1010 & lon Sputter (Au) E3P, Japan \\
\hline
\end{tabular}


the tail vein, 2 times per rat. From the $10^{\text {th }} \mathrm{W}$, rats were fed with $1 \mathrm{ml} / 100 \mathrm{~g}$ distilled water every day until the end of the $17^{\text {th }} \mathrm{W}$.

Treatment of rats in the low dose treatment group: at the end of the $9^{\text {th }} \mathrm{w}$, empty fasting water was poured into a clean metabolism cage and the outlet of the metabolism rat cage was closed with filter paper. The urine of the rats was collected for $24 \mathrm{~h}$ and the urine volume was recorded to quantify the urine protein in the urine of the rats for $24 \mathrm{~h}$. One rat was randomly selected from the three model groups and the normal group. Observed Hematoxylin and eosin staining (HE staining) and immunofluorescence staining of the selected rats were used to confirm the successful establishment of the IgA nephropathy model and continue the subsequent relevant experiments. After successful modeling, all rats were randomly divided into 4 groups according to the method provided in the value: normal group, model group, low dose treatment group and high dose treatment group. The method of administration of rats in each group: the normal control rats were given distilled water every day, which was different according to body weight. Each $1 \mathrm{ml} / 100 \mathrm{~g}$ body weight lasted a total of $8 \mathrm{w}$. Rats in the model control group: similarly, rats were given distilled water at $1 \mathrm{ml} / 100 \mathrm{~g}$ body weight every day and the whole process lasted $8 \mathrm{w}$. The low dose treatment group was fed with xiyin liangxue decoction $(1.4 \mathrm{~g} / \mathrm{ml})$ at a daily weight of $1 \mathrm{ml} / 100 \mathrm{~g}$ for $8 \mathrm{w}$. In the high dose treatment group, xiyin liangxue decoction $(2.8 \mathrm{~g} / \mathrm{ml})$ was given to rats at a standard of $1 \mathrm{ml} / 100 \mathrm{~g}$ body weight for $8 \mathrm{w}$.

Collection of experimental specimens: urine specimens of all experimental rats were collected on the weekends of the $9^{\text {th }}$ and $17^{\text {th }} \mathrm{W}$ of the experiment. The urine of rats was collected separately for $24 \mathrm{~h}$ in advance. During this period, the rats were prohibited from eating and the rats were unable to stop drinking water to carry out $24 \mathrm{~h}$ urine protein quantitative detection.

Preparation of rat blood samples: at the end of the $9^{\text {th }} \mathrm{W}$, venous blood samples of 5 rats were drawn; serum was separated after the extraction and stored in a refrigerator at $-20^{\circ}$ for use. After the rats were sacrificed, kidney samples of all rats were collected and stored in a liquid nitrogen tank for later use. At w 17 , all remaining experimental rats were sacrificed and serum samples of the rats were collected and placed in a $4^{\circ}$ refrigerator for further blood testing. Then, a part of the collected kidney cortex of the rat was taken out and fixed in a $2.5 \%$ glutaraldehyde solution at $4^{\circ}$ overnight and then fixed and stored in a $-20^{\circ}$ refrigerator for later use. The rest of the collected rat kidney cortex is stored in a liquid nitrogen tank and then used for western blotting and real-time polymerase chain reaction (RTPCR) detection.

Detection of biochemical indicators and related methods: in the laboratory of the Experimental Animal Center, all the experimental rats were tested for $24 \mathrm{~h}$ urine protein quantification, including blood purity egg white albumin (ALB), blood creatinine (Scr), blood urea nitrogen (BUN) and alanine aminotransferase (ALT), AST. Serum detection methods: using immunofluorescence staining, HE staining, immunofluorescence staining and HE staining and other different staining methods for detection, all staining tests are prepared in the pathology room of a laboratory animal center and the electron microscope is used to observe the rat kidney podocytes after detection ultrastructure. Specific steps for specimen preparation: remove multiple tissues from the renal cortex of experimental rats and then proceed as follows: first rinse, then pour out the fixative, rinse with phosphate buffer sample $\mathrm{pH}$ 7.0 E times, each time $15 \mathrm{~min}$, then fix the sample with osmotic acid for $1 \mathrm{~h}$ or $2 \mathrm{~h}$, then treat the sample with $100 \%$ ethanol for about $20 \mathrm{~min}$ and finally treat the sample with pure acetone for about 20 min. Permeability gradient method of embedding medium: treat samples with medium embedded in acetone mixture $(\mathrm{v} / \mathrm{v}=1 / 1)$ for $1 \mathrm{~h}$, then treat samples with medium embedded in acetone mixture $(\mathrm{v} / \mathrm{v}=3 / 1)$ for approximately $3 \mathrm{~h}$, embed the pure permeability sample in the permeable sample overnight. In this step, the sample is transferred to a dry sample of a new tube, the new tube is placed in a pure bag agent, and finally the sample is embedded by the permeation method. The obtained 50-70 $\mathrm{nm}$ samples were continuously sliced in an ultra-thin microtome. After slicing, a strip composed of 6-10 consecutive ultra-thin slices was installed on the copper mesh.

Western blot detection of protein in kidney tissue: detection of protein expression in kidney tissue in a biochemical laboratory. Real-time fluorescence quantitative PCR was used to detect the expression level of podocin messenger RNA (mRNA) in kidney tissue: the expression level of podocin mRNA in kidney tissue was detected in a biochemical laboratory. Statistical analysis of gene expression differences was performed by real-time PCR. Note: each sample was repeated 3 times, and the relative expression level of each gene was analyzed within the range of CT internal reference gene-C target gene. 
Statistical analysis of experimental data: all data are calculated and compared with SPSS15.0 statistical software and paired comparison is performed by Students-Newman-Keels (SNK) Q test, with $\mathrm{p}<0.05$ having statistical significance.

\section{RESULTS AND DISCUSSION}

First, the performance of experimental IgA nephropathy rats was analyzed. The study found that the rats in the model group began to have different degrees of depression, dark hair, untidy and curly hair and a few rats showed hair loss symptoms and other adverse symptoms. After $6 \mathrm{w}$ of injection of lipopolysaccharide in the tail vein of rats, the rats had different degrees of depression, shaggy hair, yellowish and dull color, markedly reduced activity, decreased appetite, loose stools and no increase or decrease in body weight. There was one dead rat. After the second injection of LPS into the tail vein of rats at the $8^{\text {th }} \mathrm{w}, 3$ rats died and the mortality rate was $10 \%$ during the modeling period. At the end of w 9, one rat was randomly selected from the three treatment groups and the normal control group. The selected 4 rats were sacrificed and a part of the kidney tissues of the rats were taken for observation HE staining and immunofluorescence staining, to prove the success of detecting the IgA nephropathy model. The performance of the three treatment groups: the symptoms of the above mentioned symptoms in the treatment groups were gradually relieved 1 to $2 \mathrm{w}$ after the administration and the performance of the control group of rats: the symptoms of the model group gradually improved relief, in which one rat in the model group died at the $12^{\text {th }} \mathrm{W}$ and one rat died in each low dose treatment group. The specific mortality of rats in each treatment group is shown in Table 2.

The pathogenesis of $\operatorname{IgA}$ nephropathy is still unclear. In general, scholars mainly believe that mucosal immune deficiency, decreased ability to clear immune complexes, cytokines and genetic factors may be potential mechanisms that lead to the progress of IgA nephropathy, but the evidence for these ideas is quite limited. Studies have found that in patients with IgA nephropathy, the increase in structurally abnormal IgA1 polymer will stimulate the activation of the complement system and the occurrence of inflammatory reactions and may be an important factor leading to the progression of IgA nephropathy. At the same time, it was found that the activation of the mannose-binding lectin (MBL) pathway in the complement system may have certain differences in liver and kidney function and urine volume in each group of rats. Compared with the normal group, the MBL level in the model group was significantly increased $(p<0.05)$, compared with the model group, the Scr level of normal group rats decreased $(p<0.05)$ and the MBL level of rats also increased $(p<0.05)$. After the experimental rats were treated with xiyin liangxue decoction, proteinuria supplementation in the low-dose and high-dose treatment groups was improved (both $\mathrm{p}<0.05$ ). The number of urine red blood cells and urine MBL expression in the three treatment groups. They also all declined (all $\mathrm{p}<0.05$ ), the specific data is shown in fig. 1.

From the data in fig. 1, it can be seen that after treatment of IgA nephropathy rats with xiyin liangxue decoction, the condition of the rats has improved significantly, the renal function of the high-dose treatment group has been significantly improved and the expression levels of ALT and AST have increased by $19 \%, 5 \%$, ALB, globulin (GLB) expression increased by $19 \%$ and $25 \%$.

The activation of the MBL pathway in the complement system may play a very important role in the progression mechanism of IgA nephropathy. Some studies have shown that systemic and local complement activation are important factors that lead to the spread of renal damage and enhanced inflammatory response. As an activator of the lectin pathway, MBL has been reported as a potential regulator of a variety of diseases, including ischemia, reperfusion renal injury, diabetic nephropathy IV, systemic lupus erythematosus nephritis, rheumatoid arthritis and respiratory diseases and severe diseases such as coronary heart disease. Some studies have found positive results of immunohistochemical staining for MBL in IgA and a large amount of MBL accumulation has been found in patients with more severe clinical manifestations of IgA nephropathy. Therefore, this

TABLE 2: DEATH OF RATS IN EACH GROUP DURING TREATMENT PERIOD

\begin{tabular}{|c|c|c|c|c|}
\hline Group & Total case & Experimental case & Death case & Death rate \\
\hline Normal group & 10 & 9 & 0 & 0 \\
\hline Model group & 10 & 10 & 1 & $10 \%$ \\
\hline Low-dose treatment group & 10 & 9 & 1 & $10 \%$ \\
\hline High-dose treatment group & 10 & 9 & 0 & 0 \\
\hline
\end{tabular}




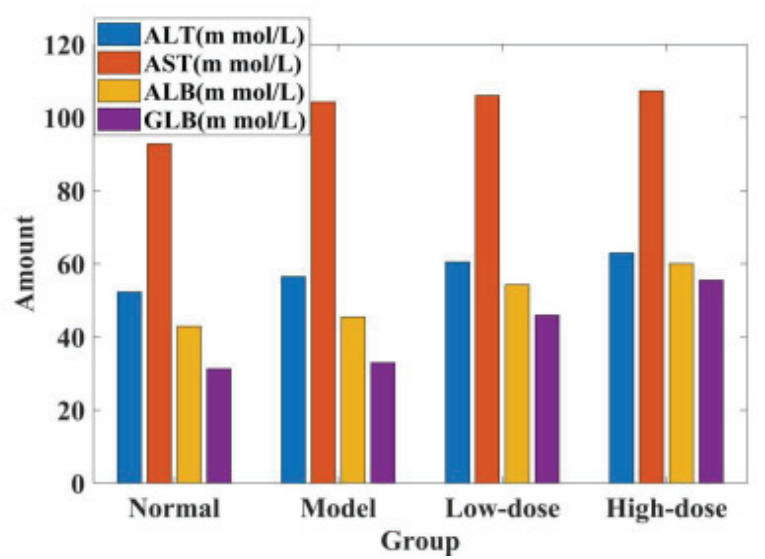

Fig. 1: Comparison of renal function and urine output of rats in each group

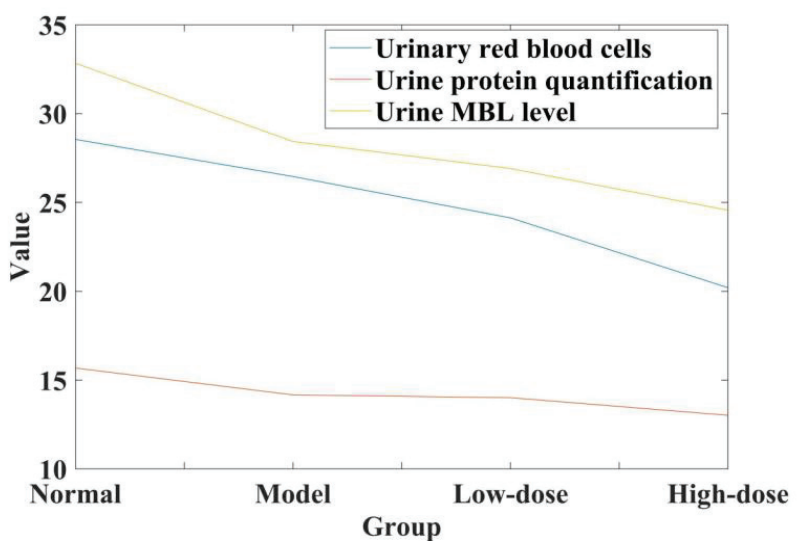

Fig. 2: Changes in urine erythrocyte number, urine protein quantity and urine MBL level of rats in each group

study compared the urine red blood cell count of each group of rats in detail, quantified urine protein and MLB levels; the specific data is shown in fig. 2.

As can be seen from the data in fig. 2, compared with the normal group, the urine red blood cells and urine protein of the model group increased significantly $(\mathrm{p}<0.05)$.

MBL also increased at the same time $(p<0.05)$. After the experimental rats were treated with xiyin liangxue decoction, the proteinuria of the low dose treatment group and the high dose treatment group were improved $($ all $p<0.05)$ and the number of urine red blood cells and urine MBL in all three treatment groups. The effect was the most obvious in the high dose treatment group. The number of urine red blood cells, urine protein and urine MBL decreased by $29 \%, 13 \%$ and $25 \%$ respectively.

TCM mainly uses a combination of syndrome differentiation and treatment, combined with syndrome differentiation and syndrome differentiation. It also shows its related advantages and characteristics in the treatment of $\operatorname{IgA}$ nephropathy. IgA nephropathy can be classified into "blood syndrome", "urinemic blood", "edema", "back pain" and other categories of Chinese medicine according to its clinical characteristics. In the acute stage, the disease usually moves the bladder down from the evils of wind heat and damp heat, heat hurts the blood collaterals or gathers heat and humid and stasis the blood. If it persists for a long time, qi damage will lead to spleen and kidney qi deficiency, qi and yin deficiency, liver and kidney yin deficiency, etc. In the course of the disease, the kidney is the main lesion center and the liver and spleen can be involved. The research group combined with the experience of the national famous Chinese medicine professor Zhu Liangshan in the treatment of chronic kidney disease, formulated "Xiyin Liangxue Decoction", based on this prescription, it was added to the clinical treatment of IgA nephropathy and achieved obvious clinical efficacy urinary erythrocytes and proteinuria of patients with nephropathy have a good improvement effect. After treatment, the functional changes of renal progenitor cells in rats in each group are shown in fig. 3.

As can be seen from fig. 3, Xiyin liangxue decoction has a good effect on clinical treatment of IgA nephropathy. The urine volume of the high dose treatment group was significantly reduced by $13 \%$ and the expressions of BUN and Scr increased by $22 \%$ and $8 \%$ respectively.

The most basic lesions of glomeruli in IgA nephropathy are located in the mesangial area. Mesangial cells and different degrees of interstitial hyperplasia can be accompanied by different amounts, uneven distribution and different morphological densities. The deposition of hemispheres in the mesangial area of the glomeruli is a characteristic change of the disease. In addition, there are still intraepithelial or subepithelial deposits, the basement membrane shrinks, thins, dissolves or is exposed and is accompanied by a large amount of

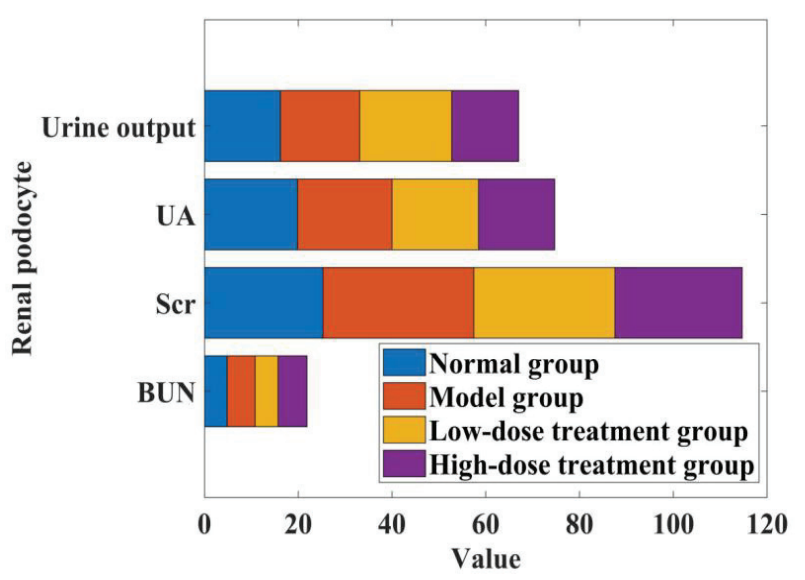

Fig. 3: Comparison of renal podocyte function in various groups 


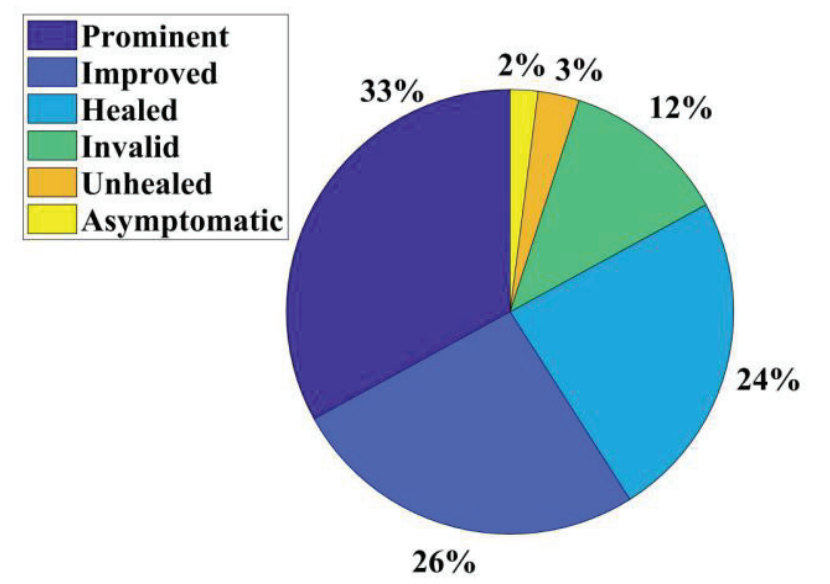

Fig. 4: The experimental performance of IgA nephropathy rats

proteinuria, usually accompanied by foot fusion. When the podocyte is injured, its morphology will change, mainly manifested as podocyte vacuole degeneration and swelling, necrosis, abscess, podocyte fusion, podocyte adhesion, shape inversion, fusion into a high electron density dam and attach to outside of GBM, podocyte proliferation, foam cell formation, etc. Under normal circumstances, under the electron microscope, testicular stromal cells can maintain their special finger morphology. When the damage to the special morphology of podocytes will change, foot process retraction may occur and the primary process becomes shorter and thinner. The foot or diffuse fusion even disappears and the disordered arrangement between the steps narrows the filter gap between the steps, even considering expansion on the basement membrane. The study found that the degree of foot process fusion was consistent with increased glomerulosclerosis, selective filtration damage and reduced GFR in IgA nephropathy, suggesting that podocyte damage is a symptom of increased IgA nephropathy. The degree of foot process fusion has a certain correlation with IgA nephropathy proteinuria and glomerulosclerosis. In the wide foot process fusion group, severe glomerulosclerosis occurs without obvious changes in the foot process, and urine protein $>2.0 \mathrm{~g} / \mathrm{d}$ foot. The incidence of sudden fusion urine protein $<2$. The $0 \mathrm{~g} / \mathrm{d}$ group increased significantly, suggesting that rapid foot process fusion can be used as a sign of the progress of glomerulosclerosis in IgA nephropathy. The ultrastructural pathology of rats treated with xiyin liangxue decoction is shown in fig. 4 .

As can be seen from fig. 4 , after treatment with xiyin liangxue decoction, $24 \%$ of the experimental IgA rats have been cured, $33 \%$ have significantly improved, $26 \%$ of the situation has been improved, $12 \%$ of the rats have no obvious improvement and $2 \%$ have no symptoms $3 \%$ not cured.

IgA nephropathy is a common primary glomerular disease. The individual difference is relatively large, and the symptoms are mainly manifested as hematuria, which may be associated with or without varying degrees of proteinuria, hypertension and impaired renal function. In China, the proportion of patients with IgA nephropathy in primary glomerular disease is about $40 \%-45 \%$. The unique immune regulation function of TCM is the main mechanism for its therapeutic effect. Xiyin liangxue decoction is a well-known TCM experience in treating chronic kidney disease. Its efficacy is mainly to nourish qi and nourish yin, strengthen the spleen and kidney and it is good for the treatment of IgA nephropathy.

The results of the study showed that after treatment of IgA nephropathy rats with Xiyin liangxue decoction, the renal function of the high-dose treatment group was significantly improved, the expression levels of ALT and AST increased by $19 \%$ and $5 \%$ and the expression levels of ALB and GLB were respectively increased by $19 \%$ and $25 \%$. Compared with the normal group, the urinary erythrocytes and urinary protein in the model group increased significantly $(\mathrm{p}<0.05)$ and urine $\mathrm{MBL}$ also increased $(p<0.05)$. In addition, the number of urinary red blood cells and urine MBL in the treatment group also decreased. The effect of the high-dose treatment group was the most obvious. The number of urinary red blood cells, urine protein and urine MBL decreased by $29 \%, 13 \%$ and $25 \%$ respectively.

The study in this article shows that Xiyin liangxue decoction has a good effect on clinical treatment of IgA nephropathy. The urine volume of the high-dose treatment group was significantly reduced by $13 \%$ and the expressions of BUN and Scr increased by $22 \%$ and $8 \%$ respectively. After the treatment of Xiyin liangxue decoction, $24 \%$ of the experimental IgA rats were cured, $33 \%$ were significantly improved, $26 \%$ of the situation was improved, $12 \%$ of the rats were not significantly improved, $2 \%$ were asymptomatic and $3 \%$ were not cured.

\section{Authors' contributions:}

All authors contributed equally to this work.

\section{Conflict of interests:}

The authors declared no conflicts of interest. 


\section{REFERENCES}

1. Li HQ, Wei JJ, Xia W, Li JH, Liu AJ, Yin SB, et al. Promoting blood circulation for removing blood stasis therapy for acute intracerebral hemorrhage: a systematic review and metaanalysis. Acta Pharmacol Sin 2015;36(6):659-75.

2. Sha H, Guo S, Liu Y, Zhao J. Combination of Qinzhu Liangxue Decoction and Acitretin on the Treatment of Psoriasis Vulgaris: A Randomized Controlled Trail. Int J Clin Exp Med 2016;9(4):7256-64.

3. Timmermans SA, van Paassen P, Cohen Tervaert JW. Recent advances in the understanding of immune-mediated nephrotic syndrome: diagnostic and prognostic implications. Expert Rev Clin Immunol 2015;11(4):489-500.

4. Nagata R, Sagawa A, Han KH, Shimada K, Kato K, Sato T, et al. Effects of Cooked Rice Flours on Cecal Fermentation in Rats. Nippon Eiyo Shokuryo Gakkaishi 2017;70(2):61-7.

5. Han ZY, Wang F, Wang C, Du LN, Zhao HX. Protective Effect of Shenqi Pills on Male Reproductive Dysfunction in Natural Aging Rats and Its Mechanism. Chin J New Drug 2018;27(24):2933-8.

6. Turkez H, Sönmez E, Di Stefano A, Mokhtar YI. Health risk assessments of lithium titanate nanoparticles in rat liver cell model for its safe applications in Nano pharmacology and Nano medicine. Cytotechnology 2016;68(2):291-302.

7. Shinoda Y. Cathodic Protection System and coating System. Pigment Paint Printing Ink 2015;88(1):338-46.

8. Zhang L, Wang XZ, Li YS, Zhang L, Hao LR. Icariin ameliorates IgA nephropathy by inhibition of nuclear factor kappa b/Nlrp3 pathway. FEBS Open Bio 2017;7(1):54-63.

9. Jing LI, Liu Y, Sang X, Wang T, Liu J. Therapeutic effect of Turkish galls extract on IgA nephropathy model rats. Chin J Pharmacol Toxicol 2017;31(6):547-52.

10. Iwatani H, Nagasawa Y, Yamamoto R, Iio K, Mizui M, Horii A, et al. CD16+ CD56+ cells are a potential culprit for hematuria in IgA nephropathy. Clin Exp Nephrol 2015;19(2):216-24.

11. Liu D, Liu Y, Chen G, He L, Tang C, Wang C, et al. Rapamycin enhances repressed autophagy and attenuates aggressive progression in a rat model of IgA nephropathy. Am J Nephrol 2017;45(4):293-300.

12. Sakuyama A, Ito O, Ogawa $\mathrm{Y}$, Komatsu M, Hu G, Miura T, et al. Abstract P116: Effects of Febuxostat on Blood Pressure and Renal Functions in Dahl Salt-Sensitive Rats. Hypertension 2015;66:115-6. 